\title{
CORONARY ARTERY DISEASE
}

\author{
Original Studies
}

\section{Reproducibility of Intravascular Ultrasound iMAP for Radiofrequency Data Analysis: Implications for Design of Longitudinal Studies}

\begin{abstract}
Jung Ho Heo, ${ }^{1}$ MD, Salvatore Brugaletta, ${ }^{1,2}$ MD, Hector M. Garcia-Garcia, ${ }^{1,2,3 \star}$ MD, PhD, Josep Gomez-Lara, ${ }^{1}$ MD, Jurgen M.R. Ligthart, ${ }^{1}$ BSc, Karen Witberg, ${ }_{1}^{1}$ BSc, Michael Magro, ${ }^{1}$ MD, Eun-Seok Shin, ${ }^{4}$ MD, PhD, and Patrick W. Serruys, ${ }^{1} \mathrm{MD}, \mathrm{PhD}$

Background: iMAP is a new intravascular ultrasound (IVUS) derived technique for tissue characterization using spectral analysis. Since there is a need for reproducibility data to design longitudinal studies, we sought to assess the in vivo reproducibility of this imaging technique. Methods: iMAP (40 MHz, Boston Scientific Corporation) was performed in patients referred for elective percutaneous intervention and in whom a nonintervened vessel was judged suitable for a safe IVUS analysis. Overall 20 patients with 20 non-angiographically significant lesions were assessed by two independent observers. Five of these 20 patients received an additional IMAP analysis using a new IVUS catheter and using the same catheter after its engagement and reengagement. Results: The interobserver relative difference in plaque area was $2.5 \%$. Limits of agreement for lumen, vessel, and plaque area measurements were $1.62,-2.47 \mathrm{~mm}^{2} ; 2.09,-3.71 \mathrm{~mm}^{2} ; 2.80,-3.72 \mathrm{~mm}^{2}$; respectively. Limits of agreement for fibrotic, lipidic, necrotic, and calcified measurements were $1.32,-1.44$ $\mathrm{mm}^{2} ; 0.24,-0.36 \mathrm{~mm}^{2} ; 1.50,-2.26 \mathrm{~mm}^{2} ; 0.09,-0.11 \mathrm{~mm}^{2}$; respectively. The intercatheter and intracatheter relative difference in plaque area were $0.9 \%$ and $4.1 \%$, respectively. Although the variability for compositional measurements increased using two different catheters or using the same catheter twice, the variability for compositional measurements keeps always below $10 \%$. Conclusions: Our analysis demonstrates that the geometrical and compositional iMAP analysis is acceptably reproducible. (c) 2011 Wiley Periodicals, Inc.
\end{abstract}

Key words: intravascular ultrasound; angiography; coronary; lipids

\section{INTRODUCTION}

In interventional cardiology practice, intravascular ultrasound (IVUS) has been used for quantification of the extent, severity, distribution, and morphology of coronary atherosclerosis [1]. In addition, it has also been used to evaluate the temporal effect of novel therapies on plaque progression [2-5].

Over the last years, in-vivo ultrasound tissue characterization interpreting the backscattering signal has been developed to provide some important additional information on coronary atherosclerosis [6]. Necrotic core detected by IVUS-virtual histology (VH) has been extensively studied [7] and related to clinical characteristics [8] and cardiovascular risk score [9] and associated with high risk of events in the PROSPECT trial [10]. IVUS-VH has also been used for testing the effi-
${ }^{1}$ Department of Interventional Cardiology, Thoraxcenter, Erasmus MC, Rotterdam, The Netherlands

${ }^{2}$ Thorax Institute, Department of cardiology, Hospital Clinic, Barcelona, Spain

${ }^{3}$ Cardialysis, Rotterdam BV, The Netherlands

${ }^{4}$ Division of Cardiology, Ulsan University Hospital, South Korea

Jung Ho Heo and Salvatore Brugaletta have equally contributed to this manuscript.

Conflict of interest: nothing to report.

*Correspondence to: Hector M. Garcia-Garcia, MD, PhD, Thoraxcenter, Z120, Dr. Molewaterplein 40, 3015-GD Rotterdam, Netherlands. E-mail: h.garciagarcia@erasmusmc.nl

Received 15 March 2011; Revision accepted 8 August 2011

DOI 10.1002/ccd.23335

Published online 22 November 2011 in Wiley Online Library (wileyonlinelibrary.com) 


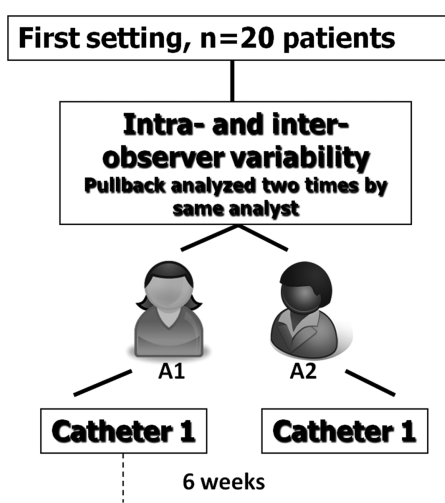

Catheter 1

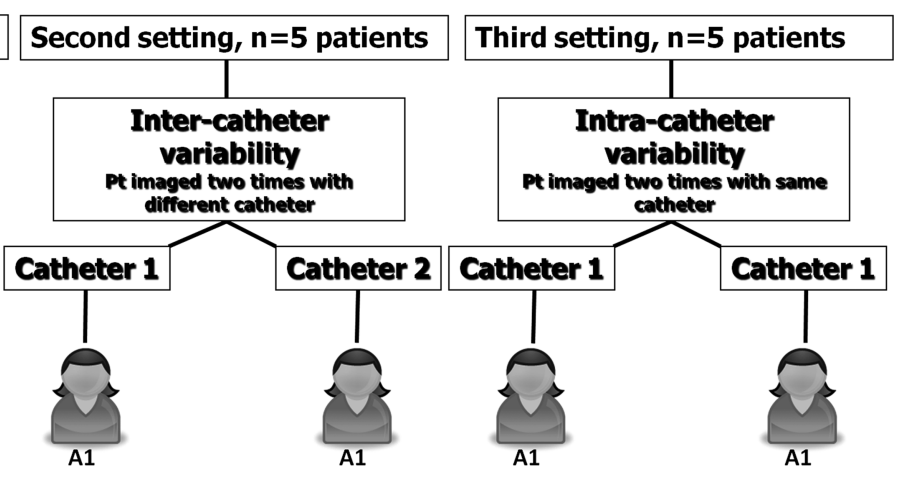

Fig. 1. Study design flow chart. cacy of novel therapies [11]. The reproducibility of this technique was shown to be acceptable for use in longitudinal studies [12].

Recently, a new intravascular ultrasound-based catheter (iMAP, Boston Scientific Corporation) for tissue interpretation has been developed, using a pattern recognition algorithm on the spectra obtained from a fast Fourier transformation and histology-derived database [13].

We sought to study the inter- and intraobserver, intracatheter and intercatheter variabilities of iMAP measurements at a single-time-point, simulating a longitudinal study. In particular, we focused on the assessment of the variability of the necrotic core because it is one of the most clinically relevant parameters.

\section{METHODS}

\section{Patient Population}

This was a prospective, investigators-driven study that sought to explore the in vivo reproducibility of spectral analysis of iMAP-IVUS (Boston Scientific Corporation). The study population consisted of consecutive patients, who were referred for elective percutaneous coronary interventions and in whom a nonintervened vessels was judged suitable for IVUS analysis of a vessel segment of at least $30 \mathrm{~mm}$. Two institutions participated in this study, Erasmus Medical Center in Rotterdam, the Netherlands and Ulsan University Hospital, Ulsan, Korea.

Exclusion criteria included the presence of severe calcification and/or vessel tortuosity and patient's hemodynamic instability.

\section{iMap-IVUS Catheter and Acquisition}

iMap IVUS uses a 40-MHz single-rotational transducer on a drive shaft and can acquire radiofrequency data continuously, when connected to Boston Scientific's
TABLE I. Baseline Clinical Characteristics $(n=20)$

\begin{tabular}{lc}
\hline Clinical characteristics & Patients $(n=20), n(\%)$ \\
\hline Age (years \pm SD) & $66.1 \pm 11.1$ \\
Male sex & $16(80)$ \\
Hypertension & $14(70)$ \\
Diabetes mellitus & $5(25)$ \\
Smoking & $3(15)$ \\
Family history of coronary disease & $8(40)$ \\
Hypercholesterolemia & $15(75)$ \\
Lipid lowering agents & $14(70)$ \\
Clinical presentation & $5(25)$ \\
Stable angina & $15(75)$ \\
ACS & $5(25)$ \\
Study vessel & \\
$\quad$ Left anterior descending & $15(75)$ \\
Left circumflex artery & $2(10)$ \\
Right coronary artery & $3(15)$ \\
\hline
\end{tabular}

$\mathrm{iLab}^{\circledR}$ Ultrasound Imaging System. As it uses a $40-\mathrm{MHz}$ transducer, iMap-IVUS has high axial resolution but displays specific artifacts such as nonuniform rotational distortion because it is a rotational catheter. iMap uses a pattern recognition algorithm on a spectra obtained from a fast Fourier transformation and a histology-derived database [13]. Using a color-code, iMap depicts fibrotic tissue as light green, lipidic tissue as yellow, necrotic tissue as pink, and calcified tissue as blue [14].

The IVUS catheters used were commercially available mechanical array catheters (Boston Scientific Corporation). The catheter probe, after intracoronary administration of isosorbide dinitrate, was advanced at least $10 \mathrm{~mm}$ distal to a clearly visible side branch and angiographic cine runs, before and during contrast injection, were performed to define the position of the IVUS catheter before the pullback was started. The catheter was withdrawn at a continuous automated pullback of $0.5 \mathrm{~mm} / \mathrm{sec}$. Subsequently, some of the patients included, underwent the same procedure using a new catheter (iMAP $40 \mathrm{MHz}$, Boston Scientific Corporation) or using the same catheter, after its 
TABLE II. Geometrical and Compositional Measurement of Matched ROI Between Different Observers $(n=20)$

\begin{tabular}{|c|c|c|c|c|}
\hline & Observer 1 & Observer 2 & Absolute $\Delta$ & Relative $\Delta(\%)$ \\
\hline \multicolumn{5}{|l|}{ Geometrical data } \\
\hline Lumen CSA $\left(\mathrm{mm}^{2}\right)$ & $5.94 \pm 1.90$ & $6.10 \pm 1.93$ & $0.15 \pm 0.19$ & 2.5 \\
\hline Lumen volume $\left(\mathrm{mm}^{3}\right)$ & $191.47 \pm 89.41$ & $195.82 \pm 93.11$ & $4.34 \pm 6.28$ & 1.7 \\
\hline Lumen max diameter (mm) & $3.49 \pm 0.63$ & $3.58 \pm 0.76$ & $0.08 \pm 0.20$ & 1.4 \\
\hline Lumen min diameter (mm) & $1.92 \pm 0.32$ & $2.01 \pm 0.27$ & $0.09 \pm 0.11$ & 4.6 \\
\hline Lumen mean diameter (mm) & $2.65 \pm 0.41$ & $2.72 \pm 0.43$ & $0.07 \pm 0.06$ & 2.4 \\
\hline Vessel CSA $\left(\mathrm{mm}^{2}\right)$ & $15.18 \pm 4.37$ & $15.15 \pm 4.35$ & $0.03 \pm 0.24$ & 0.2 \\
\hline Vessel volume $\left(\mathrm{mm}^{3}\right)$ & $486.91 \pm 206.69$ & $486.26 \pm 200.63$ & $0.65 \pm 10.09$ & 0.1 \\
\hline Vessel max diameter (mm) & $4.94 \pm 0.65$ & $5.11 \pm 0.67$ & $0.17 \pm 0.24$ & 3.1 \\
\hline Vessel min diameter (mm) & $3.48 \pm 0.84$ & $3.61 \pm 0.85$ & $0.13 \pm 0.15$ & 3.5 \\
\hline Vessel mean diameter (mm) & $4.20 \pm 0.61$ & $4.33 \pm 0.65$ & $0.13 \pm 0.10$ & 2.9 \\
\hline Plaque CSA $\left(\mathrm{mm}^{2}\right)$ & $9.23 \pm 2.83$ & $9.05 \pm 2.83$ & $0.15 \pm 0.19$ & 2.5 \\
\hline Plaque volume $\left(\mathrm{mm}^{3}\right)$ & $295.43 \pm 122.77$ & $290.44 \pm 115.62$ & $4.99 \pm 13.75$ & 1.4 \\
\hline Plaque burden $(\%)$ & $60.80 \pm 5.80$ & $59.63 \pm 6.04$ & $1.16 \pm 1.14$ & 1.9 \\
\hline \multicolumn{5}{|l|}{ Compositional data } \\
\hline Fibrotic volume $\left(\mathrm{mm}^{3}\right)$ & $151.91 \pm 62.50$ & $151.32 \pm 60.61$ & $0.60 \pm 6.90$ & 0.2 \\
\hline Fibrotic area $\left(\mathrm{mm}^{2}\right)$ & $4.59 \pm 1.84$ & $4.61 \pm 1.89$ & $0.01 \pm 0.20$ & 0.1 \\
\hline Fibrotic tissue $(\%)$ & $53.13 \pm 15.79$ & $53.66 \pm 15.61$ & $0.53 \pm 1.38$ & 1.2 \\
\hline Lipidic volume $\left(\mathrm{mm}^{3}\right)$ & $26.14 \pm 17.58$ & $25.84 \pm 16.19$ & $0.30 \pm 2.03$ & 2.0 \\
\hline Lipidic area $\left(\mathrm{mm}^{2}\right)$ & $0.78 \pm 0.49$ & $0.79 \pm 0.53$ & $0.01 \pm 0.06$ & 0.3 \\
\hline Lipidic tissue $(\%)$ & $8.46 \pm 2.10$ & $8.58 \pm 2.13$ & $0.11 \pm 0.34$ & 0.3 \\
\hline Necrotic volume $\left(\mathrm{mm}^{3}\right)$ & $112.09 \pm 81.60$ & $107.49 \pm 74.57$ & $4.60 \pm 10.20$ & 10.2 \\
\hline Necrotic area $\left(\mathrm{mm}^{2}\right)$ & $3.26 \pm 2.26$ & $3.40 \pm 2.48$ & $0.14 \pm 0.31$ & 3.5 \\
\hline Necrotic tissue $(\%)$ & $35.14 \pm 14.02$ & $35.14 \pm 13.58$ & $0.70 \pm 1.35$ & 1.9 \\
\hline Calcific volume $\left(\mathrm{mm}^{3}\right)$ & $7.33 \pm 4.16$ & $7.40 \pm 4.34$ & $0.07 \pm 0.48$ & 0.4 \\
\hline Calcific area $\left(\mathrm{mm}^{2}\right)$ & $0.22 \pm 0.13$ & $0.22 \pm 0.12$ & $0.01 \pm 0.01$ & 0.7 \\
\hline Calcific tissue $(\%)$ & $2.58 \pm 1.30$ & $2.63 \pm 1.31$ & $0.04 \pm 0.15$ & 0.7 \\
\hline
\end{tabular}

TABLE III. Geometrical and Compositional Measurement of Matched ROI Between the Same Observer $(n=20)$

\begin{tabular}{|c|c|c|c|c|}
\hline & Observer 1 (1st time) & Observer 1 (2nd time) & Absolute $\Delta$ & Relative $\Delta(\%)$ \\
\hline \multicolumn{5}{|l|}{ Geometrical data } \\
\hline Lumen CSA $\left(\mathrm{mm}^{2}\right)$ & $5.94 \pm 1.90$ & $5.93 \pm 1.89$ & $0.01 \pm 0.13$ & 0.03 \\
\hline Lumen volume $\left(\mathrm{mm}^{3}\right)$ & $191.47 \pm 89.41$ & $189.46 \pm 88.83$ & $2.02 \pm 6.48$ & 1.4 \\
\hline Lumen max diameter $(\mathrm{mm})$ & $3.49 \pm 0.63$ & $3.47 \pm 0.58$ & $0.02 \pm 0.05$ & 0.04 \\
\hline Lumen min diameter $(\mathrm{mm})$ & $1.92 \pm 0.32$ & $1.89 \pm 0.25$ & $0.03 \pm 0.07$ & 0.1 \\
\hline Lumen mean diameter $(\mathrm{mm})$ & $2.65 \pm 0.41$ & $2.68 \pm 0.43$ & $0.03 \pm 0.02$ & 0.1 \\
\hline Vessel CSA $\left(\mathrm{mm}^{2}\right)$ & $15.18 \pm 4.37$ & $15.30 \pm 4.40$ & $0.12 \pm 0.26$ & 0.7 \\
\hline Vessel volume $\left(\mathrm{mm}^{3}\right)$ & $486.91 \pm 206.69$ & $489.47 \pm 208.54$ & $2.57 \pm 9.71$ & 0.6 \\
\hline Vessel max diameter $(\mathrm{mm})$ & $4.94 \pm 0.65$ & $4.90 \pm 0.58$ & $0.04 \pm 0.27$ & 0.4 \\
\hline Vessel min diameter $(\mathrm{mm})$ & $3.48 \pm 0.84$ & $3.38 \pm 0.71$ & $0.10 \pm 0.13$ & 0.6 \\
\hline Vessel mean diameter $(\mathrm{mm})$ & $4.20 \pm 0.61$ & $4.35 \pm 0.65$ & $0.15 \pm 0.04$ & 0.6 \\
\hline Plaque CSA $\left(\mathrm{mm}^{2}\right)$ & $9.23 \pm 2.83$ & $9.37 \pm 2.82$ & $0.13 \pm 0.29$ & 1.6 \\
\hline Plaque volume $\left(\mathrm{mm}^{3}\right)$ & $295.43 \pm 122.77$ & $300.01 \pm 124.71$ & $4.58 \pm 7.32$ & 1.9 \\
\hline Plaque burden $(\%)$ & $60.80 \pm 5.80$ & $61.21 \pm 5.05$ & $0.41 \pm 1.14$ & 0.8 \\
\hline \multicolumn{5}{|l|}{ Compositional data } \\
\hline Fibrotic volume $\left(\mathrm{mm}^{3}\right)$ & $151.91 \pm 62.50$ & $153.96 \pm 63.36$ & $2.05 \pm 4.72$ & 1.5 \\
\hline Fibrotic area $\left(\mathrm{mm}^{2}\right)$ & $5.11 \pm 2.13$ & $5.19 \pm 2.17$ & $0.08 \pm 0.15$ & 1.6 \\
\hline Fibrotic tissue $(\%)$ & $53.13 \pm 15.79$ & $53.04 \pm 15.77$ & $0.09 \pm 0.95$ & 0.1 \\
\hline Lipidic volume $\left(\mathrm{mm}^{3}\right)$ & $26.14 \pm 17.58$ & $26.45 \pm 17.79$ & $0.31 \pm 0.81$ & 1.5 \\
\hline Lipidic area $\left(\mathrm{mm}^{2}\right)$ & $0.88 \pm 0.59$ & $0.89 \pm 0.60$ & $0.01 \pm 0.02$ & 1.6 \\
\hline Lipidic tissue (\%) & $8.46 \pm 2.10$ & $8.45 \pm 2.09$ & $0.01 \pm 0.09$ & 0.1 \\
\hline Necrotic volume $\left(\mathrm{mm}^{3}\right)$ & $112.09 \pm 81.60$ & $113.46 \pm 81.58$ & $1.37 \pm 4.03$ & 2.1 \\
\hline Necrotic area $\left(\mathrm{mm}^{2}\right)$ & $3.77 \pm 2.76$ & $3.83 \pm 2.77$ & $0.05 \pm 0.13$ & 1.9 \\
\hline Necrotic tissue (\%) & $35.14 \pm 14.02$ & $35.94 \pm 13.94$ & $0.11 \pm 0.84$ & 0.4 \\
\hline Calcific volume $\left(\mathrm{mm}^{3}\right)$ & $7.33 \pm 4.16$ & $7.44 \pm 4.29$ & $0.10 \pm 0.52$ & 0.7 \\
\hline Calcific area $\left(\mathrm{mm}^{2}\right)$ & $0.24 \pm 0.14$ & $0.25 \pm 0.14$ & $0.01 \pm 0.01$ & 0.8 \\
\hline Calcific tissue $(\%)$ & $2.58 \pm 1.30$ & $2.57 \pm 1.32$ & $0.01 \pm 0.17$ & 1.1 \\
\hline
\end{tabular}

Catheterization and Cardiovascular Interventions DOI 10.1002/ccd. Published on behalf of The Society for Cardiovascular Angiography and Interventions (SCAI). 

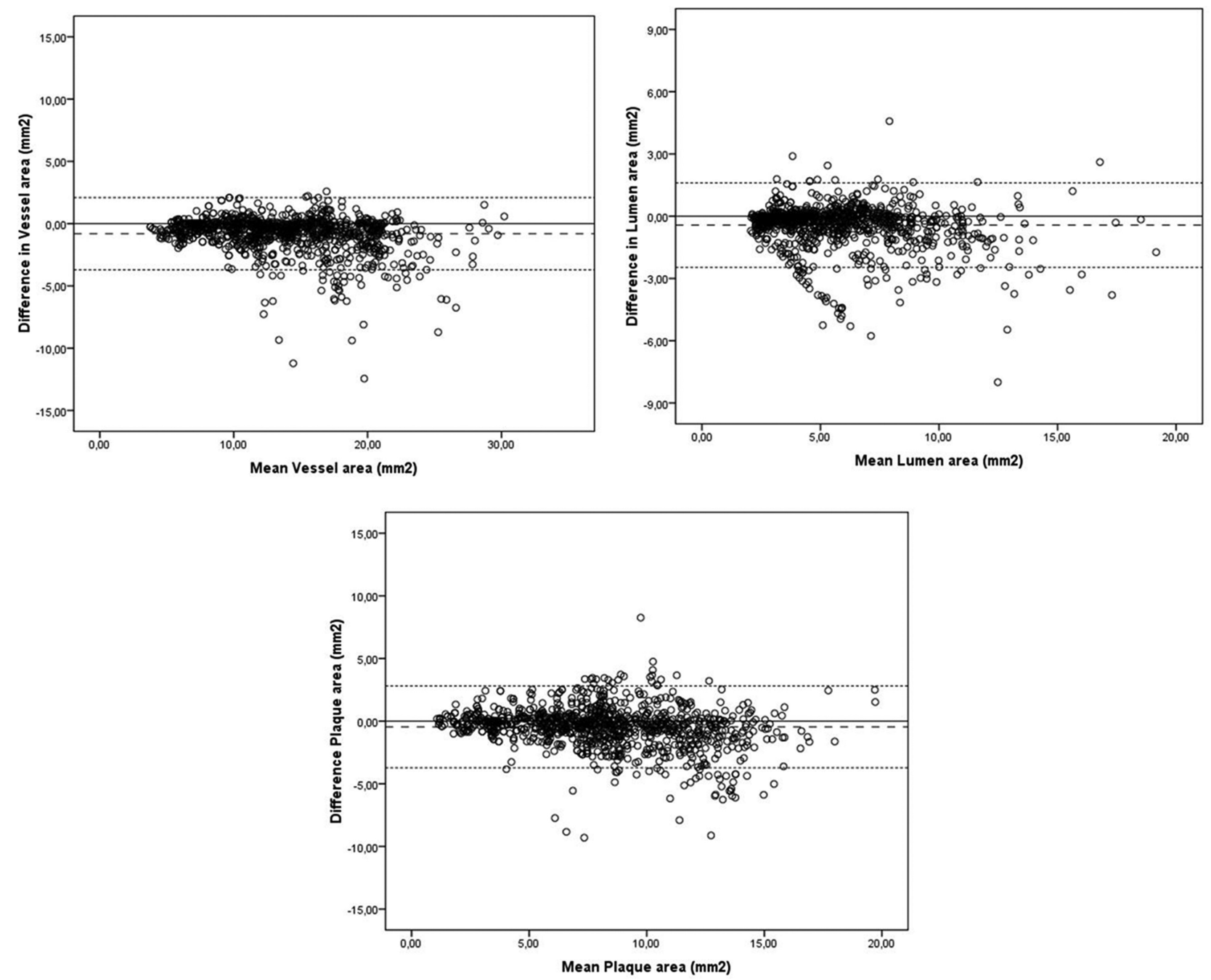

Fig. 2. Bland-Altman depicting the interobserver agreement for geometrical measurement $(n=20)$.

disengagement and reengagement, with the same side branches as landmarks. Data acquired were stored in a DVD for offline analysis.

\section{iMap-IVUS Analysis}

A region of interest (ROI) was identified between one proximal and one distal side branch. Contour detection of the lumen and the media-adventitia interface was performed by two independent experienced IVUS analysts, using a dedicated software (QIvus, Medis, Leiden, The Netherlands). One of these two IVUS analyst reanalyzed the same cases at time interval of 1 week, leading to the possibility of multiple comparisons: interobserver variability (observer 1 and $2)$; intraobserver variability (2 times observer 1$)$; intercatheter variability [observer 1 (catheter 1 and catheter 2 )]; and intracatheter variability [observer 1 (same catheter with two pullbacks)] (Fig. 1). Intracatheter, intercatheter, and interobserver agreement were calculated per ROI.

As the software used for the analysis allows the analyst to mask the shadow of the guidewire, usually detected as necrotic core, and to detect as black the signal underneath calcium, we also evaluated the variability introduced by these software features in the compositional measurements within a pullback, using the same catheter with the same geometrical contours.

The contours of the external elastic membrane (EEM) and the lumen-intima interface enclosed an area that was defined as the coronary plaque plus media area. Geometrical data were obtained for each crosssectional area and an average was calculated for each ROI. Compositional data were obtained for each ROI as volume and calculated for each cross-sectional area as percentage. 

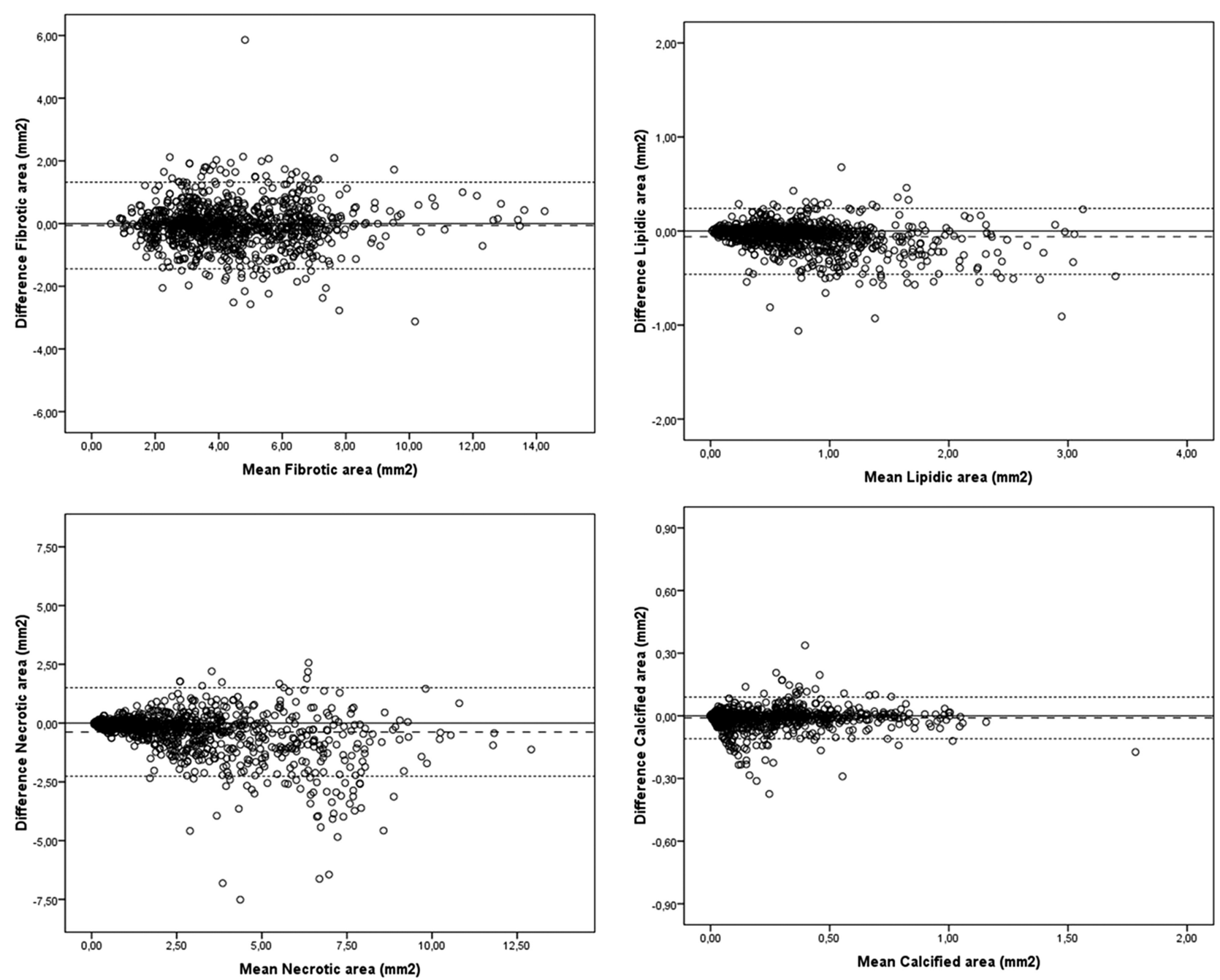

Fig. 3. Bland-Altman plots depicting the interobserver agreement for compositional measurement $(n=20)$.

\section{Statistical Analysis}

Discrete variables are presented as counts and percentages. Continuous variables are presented as mean \pm standard deviation (SD). Correlation test and BlandAltman plots were performed [16]. Limits of agreement were determined by adding two standard deviations to the mean difference for the upper limit and by subtracting two standard deviations from the mean difference for the lower limit. A two-sided $P$ value of less than 0.05 indicated statistical significance. Statistical analysis was performed with SPSS 16.0 software (SPSS, Chicago, IL).

\section{RESULTS}

\section{Patient Population}

Twenty consecutive patients with 20 nonsignificant lesions were included in the study. Baseline character- istics of the patients are shown in Table I. There were no periprocedural complications. The length of the ROI was $32.9 \pm 11.5 \mathrm{~mm}$.

\section{Inter- and Intraobserver Agreement (Tables II and III)}

For the assessment of the inter- and intraobserver agreement, a comparison between the same matched ROI analyzed by the two independent analysts or by the same analysis was done. The two datasets were merged resulting in a paired inter- and intraobserver agreement evaluation of 7,832 frames.

The relative interobserver differences regarding geometrical measurements were acceptable. Narrow limits of agreement were found between observers for geometrical (limits of agreement for lumen, vessel, and plaque area measurements of $1.62,-2.47 \mathrm{~mm}^{2} ; 2.09$, $-3.71 \mathrm{~mm}^{2} ; 2.80,-3.72 \mathrm{~mm}^{2}$; respectively). With 
TABLE IV. Geometrical and Compositional Measurement of Matched ROI Between Two Catheters $(n=5)$

\begin{tabular}{|c|c|c|c|c|}
\hline & Catheter 1 & Catheter 2 & Absolute $\Delta$ & Relative $\Delta(\%)$ \\
\hline \multicolumn{5}{|l|}{ Geometrical data } \\
\hline Lumen CSA $\left(\mathrm{mm}^{2}\right)$ & $8.73 \pm 2.68$ & $8.80 \pm 2.66$ & $0.08 \pm 0.15$ & 1.6 \\
\hline Lumen volume $\left(\mathrm{mm}^{3}\right)$ & $206.54 \pm 82.75$ & $204.26 \pm 85.68$ & $2.27 \pm 4.83$ & 1.7 \\
\hline Lumen max diameter (mm) & $5.06 \pm 0.78$ & $5.18 \pm 0.86$ & $0.12 \pm 0.17$ & 2.2 \\
\hline Lumen min diameter (mm) & $3.74 \pm 0.56$ & $3.64 \pm 0.66$ & $0.10 \pm 0.16$ & 3.2 \\
\hline Lumen mean diameter $(\mathrm{mm})$ & $4.40 \pm 0.57$ & $4.39 \pm 0.58$ & $0.00 \pm 0.02$ & 0.1 \\
\hline Vessel CSA $\left(\mathrm{mm}^{2}\right)$ & $15.53 \pm 4.09$ & $15.52 \pm 4.14$ & $0.00 \pm 0.10$ & 0.1 \\
\hline Vessel volume $\left(\mathrm{mm}^{3}\right)$ & $471.30 \pm 178.00$ & $470.93 \pm 178.32$ & $0.36 \pm 2.74$ & 0.1 \\
\hline Vessel max diameter (mm) & $5.06 \pm 0.78$ & $5.18 \pm 0.86$ & $0.12 \pm 0.17$ & 2.2 \\
\hline Vessel min diameter (mm) & $3.75 \pm 0.48$ & $3.91 \pm 0.59$ & $0.16 \pm 0.27$ & 3.7 \\
\hline Vessel mean diameter $(\mathrm{mm})$ & $4.40 \pm 0.57$ & $4.39 \pm 0.58$ & $0.00 \pm 0.02$ & 0.1 \\
\hline Plaque CSA $\left(\mathrm{mm}^{2}\right)$ & $8.73 \pm 2.68$ & $8.80 \pm 2.66$ & $0.08 \pm 0.13$ & 0.9 \\
\hline Plaque volume $\left(\mathrm{mm}^{3}\right)$ & $264.76 \pm 103.17$ & $266.67 \pm 100.89$ & $1.91 \pm 4.16$ & 1.0 \\
\hline Plaque burden $(\%)$ & $55.80 \pm 7.17$ & $56.42 \pm 7.29$ & $0.61 \pm 1.01$ & 1.0 \\
\hline \multicolumn{5}{|l|}{ Compositional data } \\
\hline Fibrotic volume $\left(\mathrm{mm}^{3}\right)$ & $164.24 \pm 56.90$ & $163.25 \pm 56.62$ & $0.99 \pm 4.04$ & 0.7 \\
\hline Fibrotic area $\left(\mathrm{mm}^{2}\right)$ & $5.54 \pm 1.92$ & $5.53 \pm 1.91$ & $0.01 \pm 0.13$ & 0.4 \\
\hline Fibrotic tissue (\%) & $63.85 \pm 12.43$ & $62.79 \pm 12.43$ & $1.06 \pm 1.29$ & 1.7 \\
\hline Lipidic volume $\left(\mathrm{mm}^{3}\right)$ & $25.05 \pm 12.73$ & $25.25 \pm 11.76$ & $0.20 \pm 2.30$ & 1.2 \\
\hline Lipidic area $\left(\mathrm{mm}^{2}\right)$ & $0.84 \pm 0.43$ & $0.85 \pm 0.39$ & $0.01 \pm 0.07$ & 1.6 \\
\hline Lipidic tissue $(\%)$ & $9.41 \pm 2.63$ & $9.57 \pm 3.18$ & $0.16 \pm 0.96$ & 0.2 \\
\hline Necrotic volume $\left(\mathrm{mm}^{3}\right)$ & $72.55 \pm 53.24$ & $75.36 \pm 53.71$ & $2.81 \pm 0.61$ & 5.2 \\
\hline Necrotic area $\left(\mathrm{mm}^{2}\right)$ & $2.45 \pm 1.79$ & $2.55 \pm 1.82$ & $0.10 \pm 0.02$ & 5.5 \\
\hline Necrotic tissue $(\%)$ & $25.13 \pm 11.63$ & $26.10 \pm 11.54$ & $0.96 \pm 0.64$ & 4.2 \\
\hline Calcific volume $\left(\mathrm{mm}^{3}\right)$ & $4.11 \pm 1.85$ & $3.97 \pm 1.83$ & $0.14 \pm 0.43$ & 6.4 \\
\hline Calcific area $\left(\mathrm{mm}^{2}\right)$ & $0.14 \pm 0.06$ & $0.13 \pm 0.06$ & $0.01 \pm 0.01$ & 6.0 \\
\hline Calcific tissue $(\%)$ & $1.59 \pm 0.51$ & $1.52 \pm 0.57$ & $0.07 \pm 0.20$ & 7.3 \\
\hline
\end{tabular}

TABLE V. Geometrical and Compositional Measurement of Matched ROI Within the Same Catheter $(n=5)$

\begin{tabular}{|c|c|c|c|c|}
\hline & Catheter 1 (1st pullback) & Catheter 1 (2nd pullback) & Absolute $\Delta$ & Relative $\Delta(\%)$ \\
\hline \multicolumn{5}{|l|}{ Geometrical data } \\
\hline Lumen CSA $\left(\mathrm{mm}^{2}\right)$ & $6.52 \pm 2.21$ & $6.53 \pm 1.99$ & $0.01 \pm 0.29$ & 0.9 \\
\hline Lumen volume $\left(\mathrm{mm}^{3}\right)$ & $193.78 \pm 69.59$ & $195.08 \pm 67.87$ & $1.30 \pm 8.87$ & 1.1 \\
\hline Lumen max diameter $(\mathrm{mm})$ & $3.63 \pm 0.84$ & $3.72 \pm 0.86$ & $0.08 \pm 0.20$ & 2.3 \\
\hline Lumen min diameter (mm) & $2.15 \pm 0.29$ & $2.13 \pm 0.27$ & $0.02 \pm 0.09$ & 0.8 \\
\hline Lumen mean diameter $(\mathrm{mm})$ & $2.83 \pm 0.43$ & $2.83 \pm 0.39$ & $0.01 \pm 0.06$ & 0.3 \\
\hline Vessel CSA $\left(\mathrm{mm}^{2}\right)$ & $14.85 \pm 4.42$ & $15.21 \pm 4.31$ & $0.35 \pm 0.55$ & 2.6 \\
\hline Vessel volume $\left(\mathrm{mm}^{3}\right)$ & $492.92 \pm 111.45$ & $434.39 \pm 108.86$ & $4.47 \pm 9.02$ & 1.2 \\
\hline Vessel max diameter $(\mathrm{mm})$ & $4.98 \pm 0.80$ & $5.01 \pm 0.78$ & $0.03 \pm 0.05$ & 0.7 \\
\hline Vessel min diameter (mm) & $3.87 \pm 0.58$ & $3.93 \pm 0.54$ & $0.05 \pm 0.07$ & 1.3 \\
\hline Vessel mean diameter $(\mathrm{mm})$ & $4.30 \pm 0.62$ & $4.36 \pm 0.61$ & $0.05 \pm 0.07$ & 1.3 \\
\hline Plaque CSA $\left(\mathrm{mm}^{2}\right)$ & $8.33 \pm 3.10$ & $8.68 \pm 3.29$ & $0.34 \pm 0.41$ & 4.1 \\
\hline Plaque volume $\left(\mathrm{mm}^{3}\right)$ & $236.13 \pm 67.72$ & $239.31 \pm 65.17$ & $3.18 \pm 5.72$ & 1.7 \\
\hline Plaque burden $(\%)$ & $55.32 \pm 9.13$ & $56.04 \pm 9.02$ & $0.72 \pm 1.26$ & 1.3 \\
\hline \multicolumn{5}{|l|}{ Compositional data } \\
\hline Fibrotic volume $\left(\mathrm{mm}^{3}\right)$ & $122.11 \pm 26.17$ & $126.97 \pm 26.80$ & $4.86 \pm 2.02$ & 4.0 \\
\hline Fibrotic area $\left(\mathrm{mm}^{2}\right)$ & $4.09 \pm 0.87$ & $4.25 \pm 0.89$ & $0.16 \pm 0.06$ & 3.8 \\
\hline Fibrotic tissue $(\%)$ & $53.29 \pm 15.40$ & $53.54 \pm 17.43$ & $0.25 \pm 2.96$ & 0.1 \\
\hline Lipidic volume $\left(\mathrm{mm}^{3}\right)$ & $21.50 \pm 12.27$ & $21.87 \pm 11.66$ & $0.37 \pm 1.49$ & 1.9 \\
\hline Lipidic area $\left(\mathrm{mm}^{2}\right)$ & $0.72 \pm 0.41$ & $0.73 \pm 0.39$ & $0.01 \pm 0.05$ & 1.4 \\
\hline Lipidic tissue $(\%)$ & $8.64 \pm 3.21$ & $8.47 \pm 3.14$ & $0.17 \pm 0.48$ & 2.3 \\
\hline Necrotic volume $\left(\mathrm{mm}^{3}\right)$ & $87.87 \pm 45.03$ & $87.46 \pm 47.75$ & $0.42 \pm 7.52$ & 3.9 \\
\hline Necrotic area $\left(\mathrm{mm}^{2}\right)$ & $2.94 \pm 1.51$ & $2.93 \pm 1.60$ & $0.01 \pm 0.25$ & 5.5 \\
\hline Necrotic tissue $(\%)$ & $25.08 \pm 12.88$ & $34.33 \pm 14.62$ & $0.75 \pm 2.40$ & 5.2 \\
\hline Calcific volume $\left(\mathrm{mm}^{3}\right)$ & $7.23 \pm 2.71$ & $7.14 \pm 2.63$ & $0.09 \pm 0.73$ & 1.1 \\
\hline Calcific area $\left(\mathrm{mm}^{2}\right)$ & $0.24 \pm 0.09$ & $0.23 \pm 0.08$ & $0.01 \pm 0.02$ & 1.8 \\
\hline Calcific tissue $(\%)$ & $2.99 \pm 0.56$ & $2.85 \pm 0.75$ & $0.13 \pm 0.28$ & 5.1 \\
\hline
\end{tabular}

Catheterization and Cardiovascular Interventions DOI 10.1002/ccd.

Published on behalf of The Society for Cardiovascular Angiography and Interventions (SCAI). 

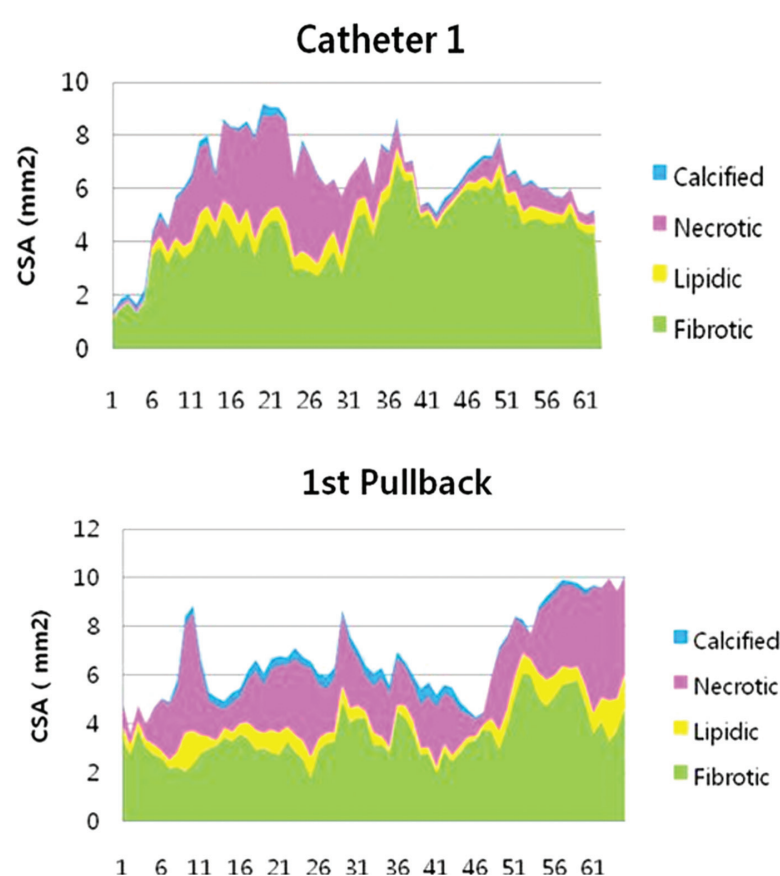

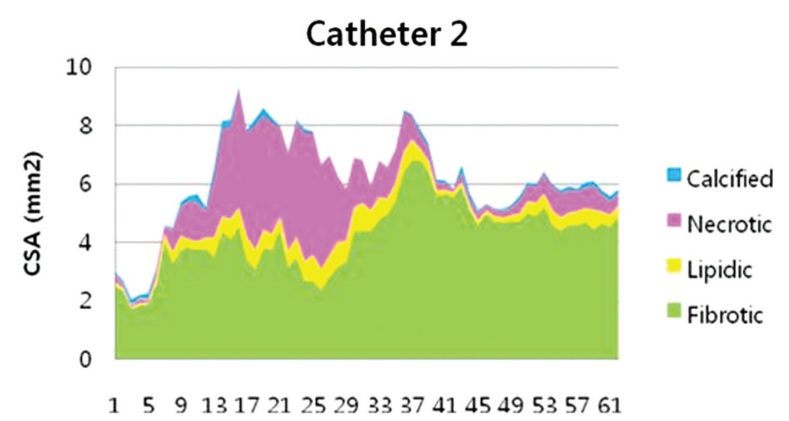

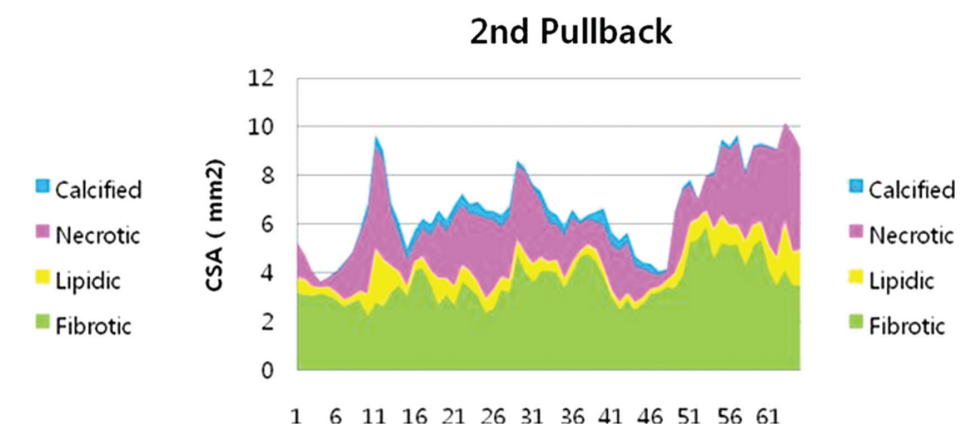

Fig. 4. Sequential plotting of a matched ROI interrogated with two different catheters (on the top) or with two different pullbacks (on the bottom). The mean cross-sectional area $(y$ axis) of each plaque component is color-coded (calcium: blue, necrotic: pink, lipidic: yellow, and fibrotic: green). These figures show examples of the impact of different catheters of pullbacks on compositional measurements. $\mathrm{ROI}=$ region of interest. 

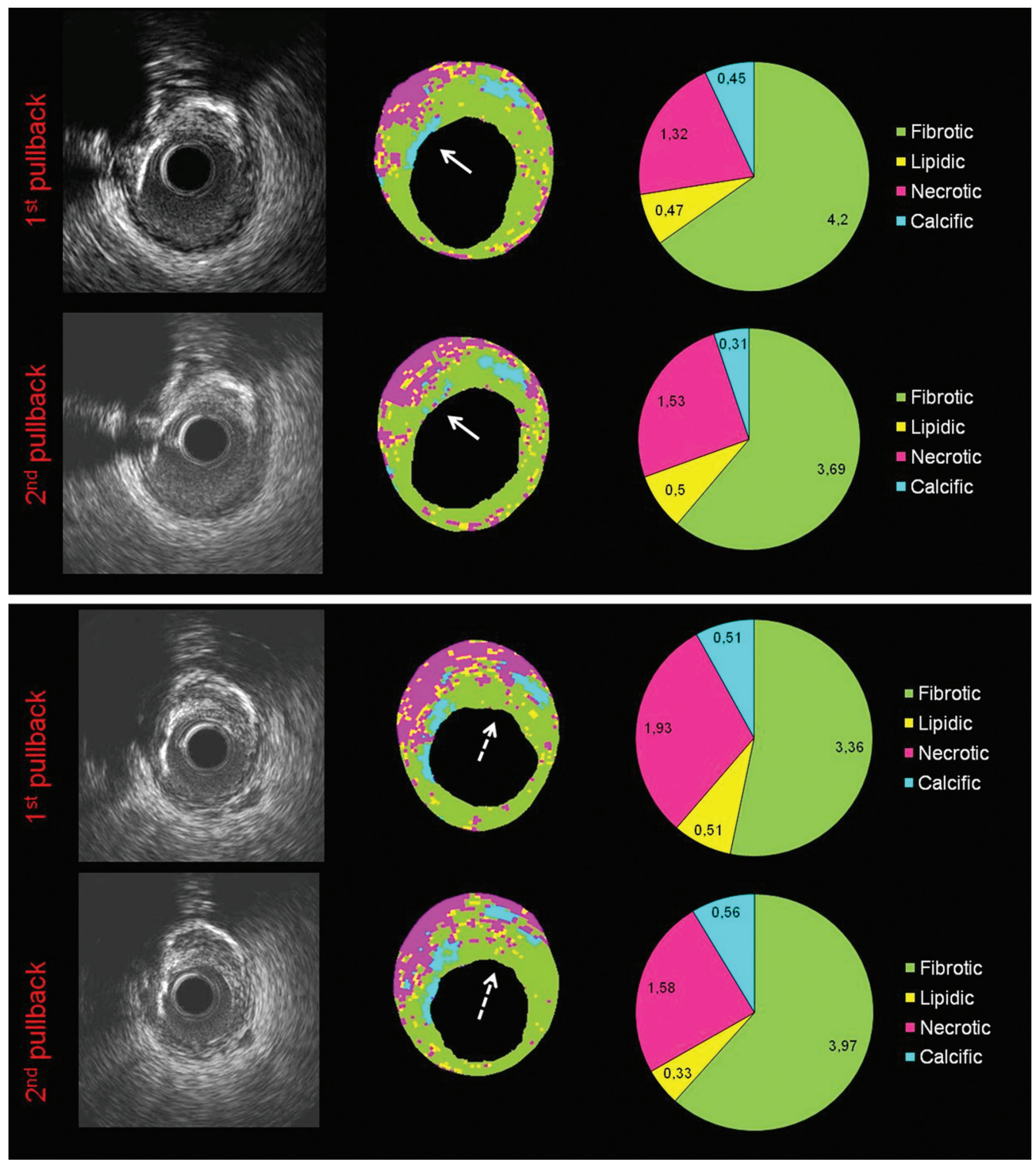

Fig. 5. Examples of the impact of two different pullbacks acquired with the same catheter on the calcium (white continuous arrows on the top panel) and necrotic core (white dotted arrows on the bottom panel) detection. Note the difference in terms of brightness between the two pullbacks.

was done. The relative intercatheter differences regarding geometrical measurements were negligible in both analyses and better than the interobserver differences, as only one observer analyzed these data. With regards to the compositional measurements, the largest relative difference was found either in necrotic or calcific tissues measurement (Fig. 4).

\section{Variability According to Various Tool of the Analysis Software (Table VI)}

Using the guidewire mask and the black pixel classification features of the software, the variability in detecting the various compositional components increases within the same pullback. In particular, the relative difference in detecting necrotic core was shown to be the highest.

\section{DISCUSSION}

The main findings of the present study are (1) iMAP geometrical measurements have an acceptable reproducibility, while compositional measurements showed lower reproducibility results; (2) in particular, necrotic core tissue had the worst reproducibility in general, while calcified tissue had acceptable reproducibility in 


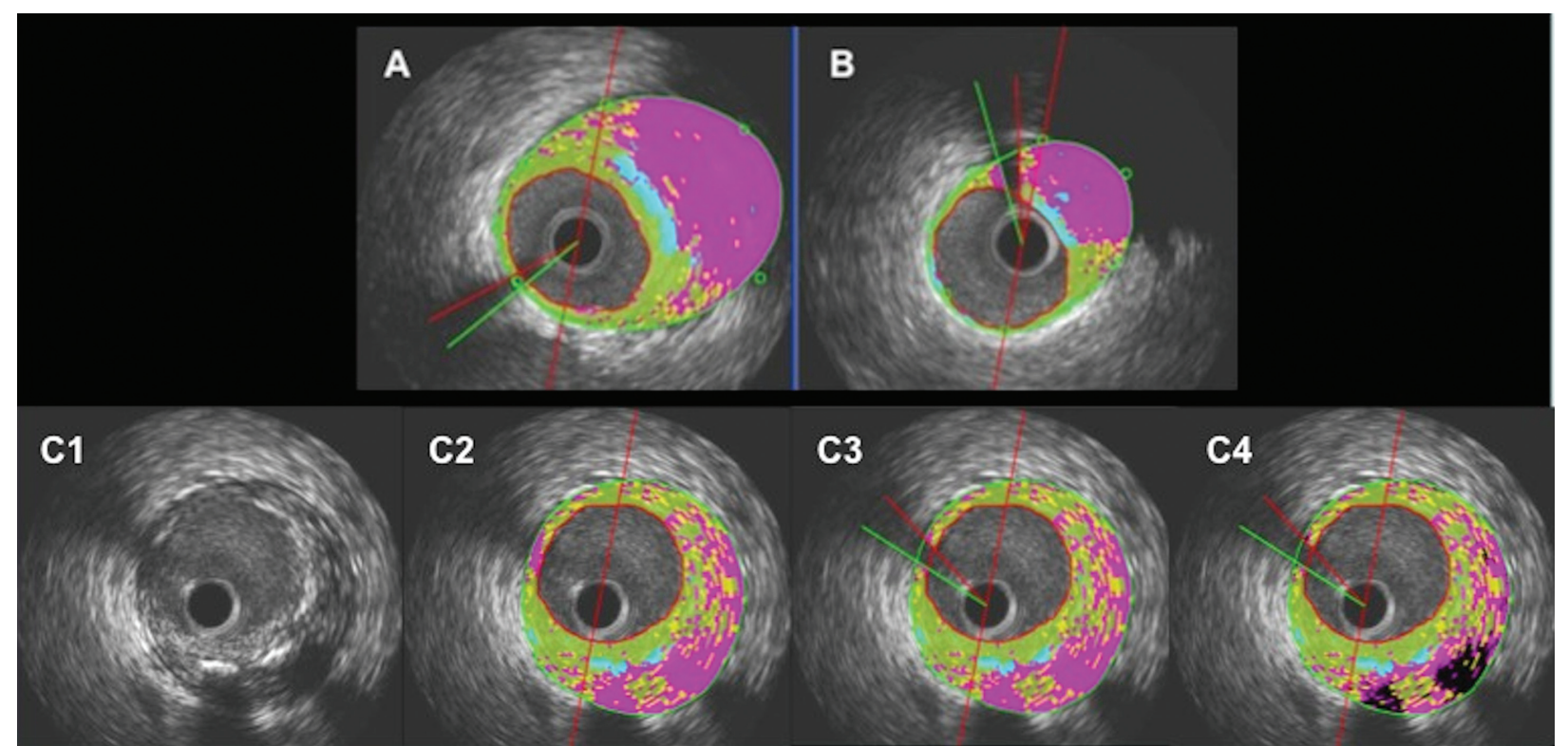

Fig. 6. The information lost due to the wire shadow is depending on where the shadow is located. If the shadow is projected on the coronary plaque, a bigger part of the plaque is excluded from the analysis (B), as compared if the shadow is projected on a part of the vessel wall without plaque (A).
The software used for the analysis allows the exclusion of the wire shadow from the tissue characterization (C1-C3) and/or the exclusion of the tissue underneath calcification, where an adequate spectral analysis is not possible due to the drop of the signal (C4). the interobserver setting, but high variability in the inter- and intracatheter variability. In addition, our study has the unique characteristic to have used two iMAP-IVUS catheters and a same iMAP-IVUS catheter twice for the evaluation of a same ROI, thus simulating a scenario of a longitudinal study.

Compositional measurements, in particular necrotic tissue, have a high variability depending on the geometrical measurements. This was an expected result, which confirms our previous findings in studying the reproducibility of the other ultrasound-based tissue technique so far available (IVUS-VH) [12]. Equal to our previous report on IVUS-VH technique, we found that interobserver and intercatheter differences are at highest $10 \%$, highly correlated and with a good agreement. Of note is that necrotic tissue showed the largest variability in the various analyses performed. This finding has an extreme importance in the design of longitudinal studies, as necrotic tissue is the most relevant and studied component of coronary plaques [10] and its variability between two observers and/or two different iMAP pullbacks has to be taken into account to evaluate its temporal change.

It is noteworthy that geometrical contours detection is important in the reproducibility of the compositional iMAP measurements. In particular, some of the observed variability in necrotic volume tissue detection $(10.2 \%)$ may be due to the interobserver variability (plaque burden difference between observers was
$1.9 \%$ ), whereas intercatheter variability contributes with $5.2 \%$ in necrotic tissue detection. Interestingly, although the intracatheter variability was $1.3 \%$ in plaque burden, the necrotic tissue detection was still high compared to the previous variabilities. One of the reasons may be due to the fact the iMAP classifies as necrotic tissue all the attenuated areas within the plaque (deeper plaque) and also the areas behind the calcium. Indeed drawing the vessel contour behind the calcium introduces important variability and the magnitude depends on the extension of the arc of calcification [17]. Calcium measurements showed a higher variability in the inter- and intracatheter analyses than in the interobserver analysis, despite a lower variability in geometrical measurements. Use of different catheters with different piezoelectric crystals or "fatigue" of the same catheter used twice could explain these findings (Fig. 5). The variability of the other iMAP plaque components also increases from the intercatheter to the intracatheter analysis. Our study with this regard may provide some thresholds over which changes are statistically significant.

It is also important to consider that iMAP-IVUS catheter is a sheath based mechanical imaging catheter and compared with electronic nonsheath based IVUSVH imaging catheter enables to obtain with more probability an uniform pullback, having that a clear importance on size and compositional measurements of atherosclerotic plaques. 
Of note is also that iMAP-IVUS catheter recognizes the shadow of the guidewire as necrotic core. Although the software helps to mask this shadow, some information is lost, depending on where the shadow is located. If the shadow is projected on the coronary plaque, a bigger part of the plaque is excluded from the analysis, as compared if the shadow is projected on a part of the vessel wall without plaque (Fig. 6). Although the reproducibility in compositional measurements is not different from that already demonstrated in the VH software, the presence of the guidewire shadow and the possibility to use the black pixel classification tool in the analysis software should be taken into account as they further increase the variability of the measurements, especially of necrotic core detection.

\section{LIMITATIONS}

The studied population was relatively small. The selection of a population of patients with nontortuous and nonseverely calcified vessels could not reproduce the same agreement of the technique in a more challenging population.

\section{CONCLUSIONS}

Our study showed that compositional measurements of iMAP are acceptably reproducible with regards to the variability in IVUS geometrical measurements. These results can aid investigators to perform power calculation for longitudinal studies using iMAP.

\section{REFERENCES}

1. Brugaletta S, Costa JR Jr, Garcia-Garcia HM. Assessment of drug-eluting stents and bioresorbable stents by grayscale IVUS and IVUS-based imaging modalities. Int $\mathrm{J}$ Cardiovasc Imaging.

2. Hirohata A, et al. Impact of olmesartan on progression of coronary atherosclerosis a serial volumetric intravascular ultrasound analysis from the OLIVUS (impact of OLmesarten on progression of coronary atherosclerosis: Evaluation by intravascular ultrasound) trial. J Am Coll Cardiol 55:976-982.

3. Takayama T, et al. Effect of rosuvastatin on coronary atheroma in stable coronary artery disease: Multicenter coronary athero- sclerosis study measuring effects of rosuvastatin using intravascular ultrasound in Japanese subjects (COSMOS). Circ J 2009; 73:2110-2117.

4. Schoenhagen $P$, et al. Determinants of arterial wall remodeling during lipid-lowering therapy: Serial intravascular ultrasound observations from the Reversal of Atherosclerosis with Aggressive Lipid Lowering Therapy (REVERSAL) trial. Circulation 2006;113:2826-2834.

5. Nissen SE, et al. Effect of very high-intensity statin therapy on regression of coronary atherosclerosis: The ASTEROID trial. JAMA 2006;295:1556-1565.

6. Nair A, et al. Coronary plaque classification with intravascular ultrasound radiofrequency data analysis. Circulation 2002;106: 2200-2206.

7. Burke AP, et al. Morphologic findings of coronary atherosclerotic plaques in diabetics: A postmortem study. Arterioscler Thromb Vasc Biol 2004;24:1266-1271.

8. Garcia-Garcia HM, et al. Synergistic effect of cardiovascular risk factors on necrotic core in coronary arteries: A report from the global intravascular radiofrequency data analysis registry. JACC Cardiovasc Imaging 2009;2:629-636.

9. Marso SP, et al. Intravascular ultrasound measures of coronary atherosclerosis are associated with the Framingham risk score: An analysis from a global IVUS registry. EuroIntervention 2009;5:212-218.

10. Stone GW, et al. A prospective natural-history study of coronary atherosclerosis. N Engl J Med 364:226-235.

11. Serruys PW, et al. Effects of the direct lipoprotein-associated phospholipase $\mathrm{A}(2)$ inhibitor darapladib on human coronary atherosclerotic plaque. Circulation 2008;118:1172-1182.

12. Rodriguez-Granillo GA, et al. Reproducibility of intravascular ultrasound radiofrequency data analysis: Implications for the design of longitudinal studies. Int J Cardiovasc Imaging 2006; 22:621-631.

13. Sathyanarayana S, Carlier S, Li W, Thomas L. Characterisation of atherosclerotic plaque by spectral similarity of radiofrequency intravascular ultrasound signals. EuroIntervention 2009; 5:133-139.

14. Shin ES, et al. In vivo findings of tissue characteristics using iMap IVUS and Virtual Histology IVUS. EuroIntervention 6: 1017-1019.

15. Shin ES, et al. Reproducibility of Shin's method for necrotic core and calcium content in atherosclerotic coronary lesions treated with bioresorbable everolimus-eluting vascular scaffolds using volumetric intravascular ultrasound radiofrequency-based analysis. Int J Cardiovasc Imaging .

16. Bland JM, Altman DG. Statistical methods for assessing agreement between two methods of clinical measurement. Lancet 1986;1:307-310.

17. Bruining $\mathrm{N}$, et al. Three-dimensional and quantitative analysis of atherosclerotic plaque composition by automated differential echogenicity. Catheter Cardiovasc Interv 2007;70:968-978. 